\title{
Depletion of Cks1 and Cks2 expression compromises cell proliferation and enhance chemotherapy-induced apoptosis in HepG2 cells
}

\author{
LINGQING LIN $^{1 *}$, ZANXI FANG $^{1 *}$, HUAYUE LIN ${ }^{1}$, HANYU YOU ${ }^{1}$, JIAJIA WANG ${ }^{1}$, \\ YUANHUI SU ${ }^{1}$, FEN WANG ${ }^{3}$ and ZHONG-YING ZHANG ${ }^{1,2}$
}

\author{
${ }^{1}$ Center for Clinical Laboratory, Xiamen University Affiliated Zhongshan Hospital, Xiamen; ${ }^{2}$ State Key Laboratory \\ of Molecular Vaccinology and Molecular Diagnostics, School of Public Health, Xiamen University, \\ Xiamen, P.R. China; ${ }^{3}$ Center for Cancer and Stem Cell Biology, Institute of Biosciences \\ and Technology, Texas A\&M Health Science Center, Houston, TX, USA
}

Received July 13, 2015; Accepted September 4, 2015

DOI: $10.3892 /$ or.2015.4372

\begin{abstract}
The present study explored the oncogenic roles of overexpressed Cks1 and Cks2 in human hepatocellular carcinoma cells. Gene expression of Cks1 and Cks2 in HepG2 cells was disrupted by siRNA or increased by cDNA transfection. Cell proliferation was assayed by CCK- 8 analysis and cell counting. Cisplatin-induced apoptosis after transfection was measured by flow cytometry using Annexin V/propidium iodide (PI) double staining. Cell cycle changes after transfection were determined by flow cytometry with PI staining. Protein levels of Akt and GSK-3 $\beta$ were measured after transfection. The results revealed that HepG2 proliferation was decreased by depletion of endogenous Cks1 or Cks2, and increased by overexpression of Cks1 or Cks2. HepG2 apoptosis increased concordantly with the decline of Cks1 or Cks2 expression. Overexpression of Cks1 or Cks2 prevented cell apoptosis. Protein levels of p-Akt and p-GSK-3 $\beta$ were downregulated after RNA interference of Cks1 or Cks2. In conclusion, Cks1 and $\mathrm{Cks} 2$ promoted proliferation and prevented apoptosis of HepG2 cells. The Akt/GSK-3 $\beta$-related PI3K/Akt signaling
\end{abstract}

Correspondence to: Professor Zhong-Ying Zhang, Center for Clinical Laboratory, Xiamen University Affiliated Zhongshan Hospital, Xiamen, Fujian 361004, P.R. China

E-mail: zhangzy1121@xmu.edu.cn

Professor Fen Wang, Center for Cancer and Stem Cell Biology, Institute of Biosciences and Technology, Texas A\&M Health Science Center, 2121 W. Holcombe Boulevard, Houston, TX 77030-3303, USA E-mail: fwang@ibt.tmc.edu

${ }^{*}$ Contributed equally

Abbreviations: PI, propidium iodide; HCC, hepatocellular carcinoma; Cks, cyclin-dependent kinase subunit; ECL, enhanced chemiluminescent; FACS, fluorescence-assisted cell sorter

Key words: cyclin-dependent kinase subunit, hepatocellular carcinoma, proliferation, apoptosis pathway may be a key signaling pathway that is involved in the regulation of cell growth and cell death.

\section{Introduction}

Hepatocellular carcinoma (HCC) represents one of the most commonly seen malignancies in the world, and has a high incident rate in China. According to the World Cancer Report of 2014 of the World Health Organization, the number of newly diagnosed cases and HCC-related death in China ranks the first in the world. It accounts for $\sim 50 \%$ of the global statistics. Since early detection of $\mathrm{HCC}$ is difficult, most HCC patients miss their optimal treatment opportunities. The efficacy of current routine chemotherapies for HCC remains unsatisfactory. It is estimated that $\sim 60-70 \%$ patients receiving radical resection have metastatic recurrence within 5 years. The 5 -year survival rate is only $7 \%$. The mechanisms underlying HCC tumorigenesis and progression remain poorly understood.

The Cks (cyclin-dependent kinase subunit) family consists of two members, Cks1 and Cks2, which are small protein molecule that are highly conserved in eukaryotic organisms. The two Cks proteins shares $81 \%$ peptide sequence homology with the yeast Cks protein (1). Emerging data show that expression of Cks proteins is closely related to the pathogenesis and progression of esophageal, prostate, gastric and colorectal cancers (2-7). We previously reported that Cks mRNA and protein expression levels in HCC tissues were elevated and correlated with cancer histopathological grading stages and AFP levels (8). Although it has been found that overexpression of the Cks family members is associated with tumorigenesis and growth, the detailed molecular mechanism by which CKS contributes to tumor cell growth and metastasis remain unclear.

Herein we further report that overexpression of Cks1 and Cks 2 by transfection increased cell proliferation, and reduced apoptosis in HepG2 cells. Consistently, depleting Cks1 or Cks2 expression by siRNA in HepG2 cells reduced cell proliferation and increased apoptosis. The results indicate that overexpressed Cks1 and Cks2 in HCC cells promote proliferation 
and survival of the cells and therefore, promotes HCC growth and progression.

\section{Materials and methods}

Materials. The human HCC HepG2 cell-line was kindly provided by the Laboratory of Hepatobiliary Surgery at the Xiamen University Affiliated Zhongshan Hospital (obtained from Shanghai Cell Bank of the Chinese Academy of Sciences, Shanghai, China). Dulbecco's modified Eagle's medium (DMEM) was obtained from Hyclone (Logan, UT, USA); fetal bovine serum (FBS) was obtained from Gibco-BRL (Gaithersburg, MD, USA); HiPerfect transfection reagent for siRNA was from Qiagen (Hilden, Germany), Lipofectamine 2000 was from Invitrogen (Carlsbad, CA, USA), TRIzol reagent for RNA extraction was obtained from Tiangen (Beijing, China). Cell Counting Kit-8 was from Dojindo (Kumamoto, Japan). RevertAid First-Strand cDNA Synthesis kits were obtained from Fermentas (Hanover, MD, USA), GoTaq Probe qPCR Master Mix used for real-time fluorescent quantitative PCR assay was obtained from Promega (Madison, WI, USA). RIPA lysis buffer was from Solarbio (Beijing, China). Cks1 antibody was obtained from Abcam (Cambridge, UK), and the Cks2 antibody was obtained from Sigma-Aldrich (St. Louis, MO, USA). Additionally, enhanced chemiluminescent (ECL) detection reagents for western blotting assays were obtained from Millipore (Billerica, MA, USA).

\section{Methods}

siRNA and overexpressing vectors. The siRNA sequences that were targeted against Cks1 and Cks2 were designed by Qiagen. The Hs-CKS1B-4 siRNA listed below were used to knock down Cks1: target sequence 5'-AAGTTTGTATGCAT TTAA-3', sense strand5'-CAUCUUUCUGAUAACAUUATT-3', and antisense strand 5'-UAAUGUUAUCAGAAAGAUGTT-3'. The Hs-CKS2-10 siRNA listed below were used to knock down Cks2: target sequence 5'-AACATCTTTCTGATAACA TTA-3', sense strand 5'-GUUUGUAUGUUGCAUUUAATT-3', and antisense strand 5'-UUAAAUGCAACAUACAAACTT-3'.

The control siRNA provided in the RNAi Human/Mouse Starter kit (Qiagen) was used for negative control. The AllStars Hs Cell Death Control siRNA was used as a cell death control. The Cks1 and Cks 2 overexpression vectors with the hygromycinresistance coding sequences were purchased from Invitrogen.

Cell culture and transfection. HepG2 cells were cultured in DMEM supplemented with $10 \% \mathrm{FBS}, 100 \mathrm{U} / \mathrm{ml}$ penicillin and $100 \mu \mathrm{g} / \mathrm{ml}$ streptomycin sulfate at $37^{\circ} \mathrm{C}$ in a $5 \% \mathrm{CO}_{2}$ incubator. Cells were cultured in 100-mm tissue culture dishes and were subcultured at a ratio of 1:3 every 2-3 days.

Transfection. HepG2 cells were inoculated in 6-well plates at a density of $2 \times 10^{5}$ cells/well just before being transfected. For gene depletion, siRNAs (300 ng) mixed with $12 \mu \mathrm{l}$ of HiPerfect transfection reagent was added to each well of the respective cultured cells. One day following transfection, the media were replaced with the fresh culture medium and the cells were cultured at $37^{\circ} \mathrm{C}$ until being used. For overexpression experiments, $2.5 \mu \mathrm{g}$ of each plasmid mixed in $6.5 \mu \mathrm{l}$ of Lipofectamine reagent were added to each well. Six hours
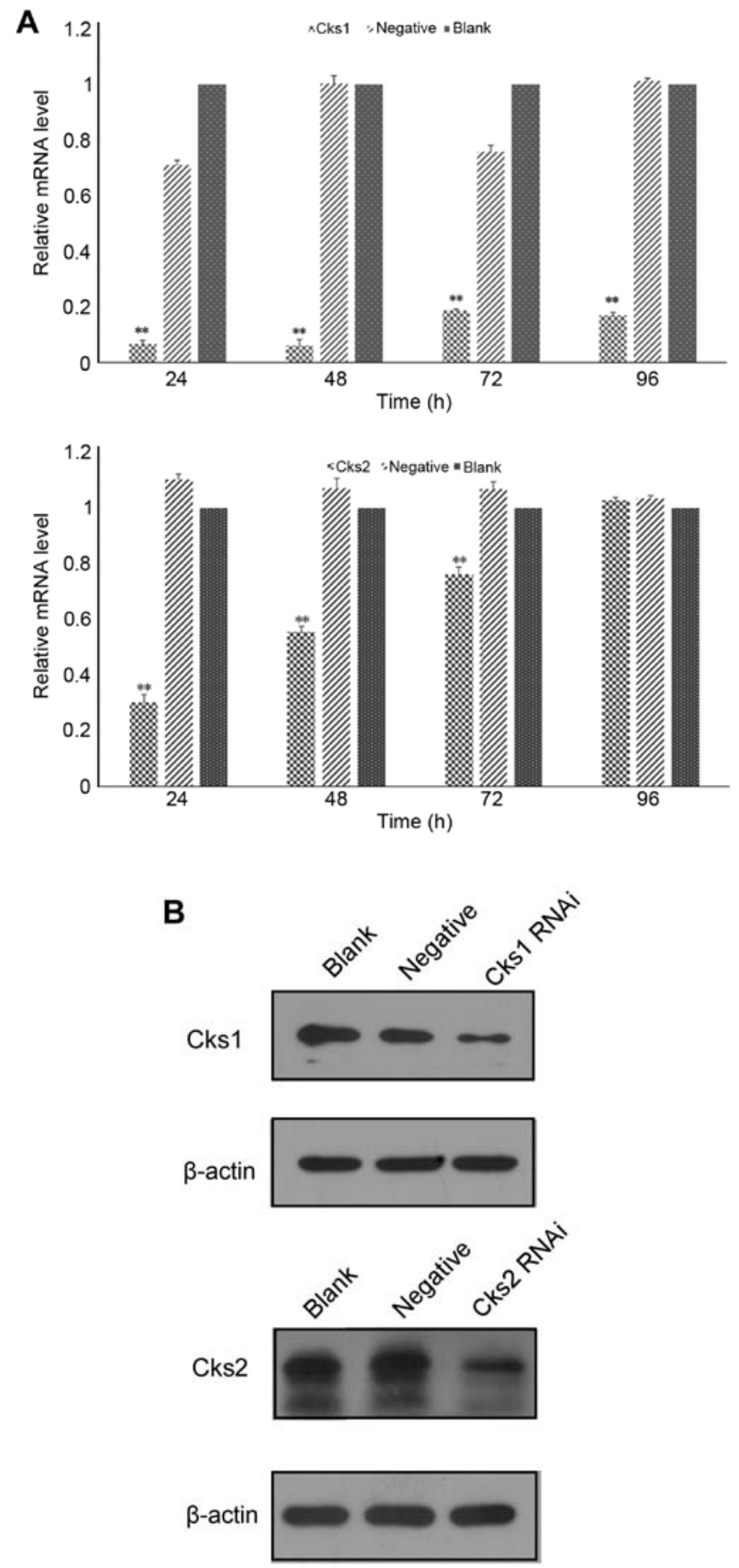

Figure 1. mRNA and protein levels of Cks1/Cks2 after transfection. (A) Cks1 and Cks2 mRNA levels after Cks1/Cks2 siRNA transfection by real-time PCR. (B) Cks1 and Cks2 protein levels examined by western blot analysis at $48 \mathrm{~h}$ after siRNA transfection. ${ }^{* *} \mathrm{P}<0.01$. The $\mathrm{Cks} 1 / \mathrm{Cks} 2$ siRNA transfection procedure resulted in significant decrease in the transcriptional and protein expression levels of Cks1 and Cks2, indicating excellent knockdown efficiency.

after the transfection, fresh culture media were added to each well. After being cultured at $37^{\circ} \mathrm{C}$ for $18 \mathrm{~h}$, the cells were subcultured at a ratio of 1:10. The medium was supplemented with $50 \mu \mathrm{g} / \mathrm{ml}$ hygromycin for selection.

Cell proliferation assays. For CCK-8 cell proliferation assay, the cells were seeded in 96-well plates at a density of $3 \times 10^{3}$ cells/ 
A

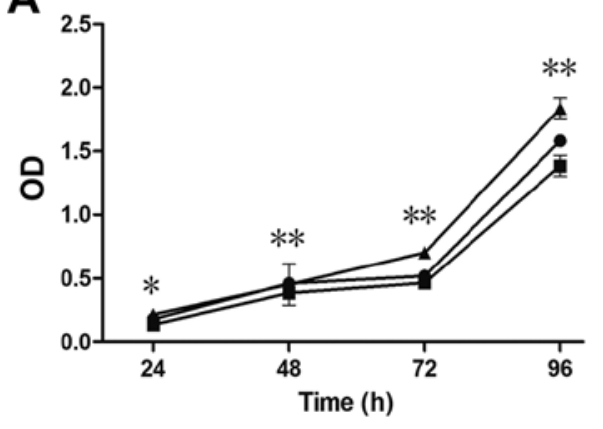

C

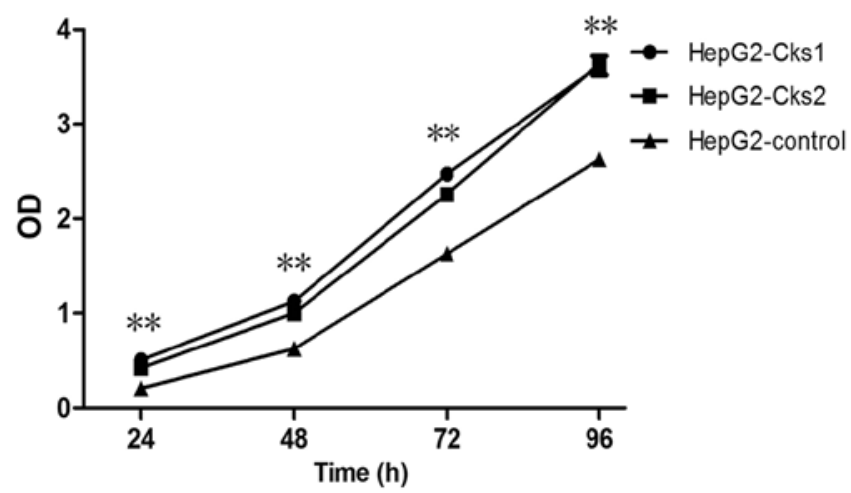

B
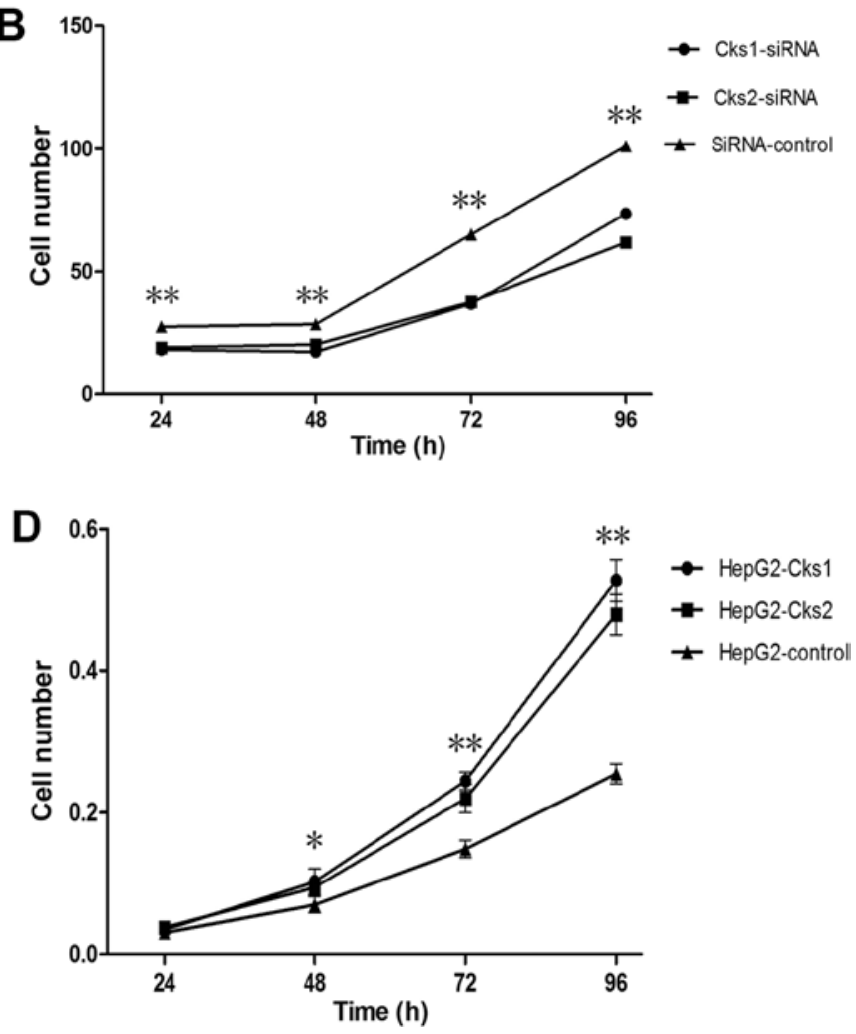

Figure 2. The effect of changes in Cks1/2 protein levels on HepG2 proliferation. (A) CCK-8 assay curve for transient siRNA transfection. (B) The cell counting curve for transient siRNA transfection. (C) CCK-8 curve for the Cks1/Cks2 overexpressed cell lines. (D) The cell counting curve for Cks1/Cks2 overexpressed cell lines. ${ }^{* *} \mathrm{P}<0.01,{ }^{*} \mathrm{P}<0.05$. Suppression of the expression of $\mathrm{Cks} 1$ and $\mathrm{Cks} 2$ protein resulted in slower proliferation.

well. The cell viability at four selected time-points $(24,48,72$ and $96 \mathrm{~h}$ ) was analyzed in quadruplicate samples by Cell Counting Kit-8 on Thermo Fisher Scientific Multiskan FC (Thermo Fisher Scientific, Waltham, MA, USA). The OD 450 values were measured and the growth curve was plotted. Alternatively, the cell number was quantitated with a Sysmex XE-5000 hemocytometer (Kobe, Japan) at four selected time-points (24, 48, 72 and $96 \mathrm{~h}$ ) in quadruplicate, and the growth curve was plotted.

For cell cycle analyses, the cells were serum-starved for $24 \mathrm{~h}$ to synchronization at the G0 phase of the cell cycle. The cells were then cultured in normal media for $48 \mathrm{~h}$ and stained with PI for fluorescent assisted flow cytometric analysis.

Assay of cell apoptosis. The cells were seeded in 6-well plates at a density of $2 \times 10^{5}$ cells/well. After cells had attached to the culture plate, cisplatin was added to the control and experimental groups at a final concentration of $3 \mu \mathrm{g} / \mathrm{ml}$ to induce apoptosis. The floating and attached cells in each well were collected at $48 \mathrm{~h}$ after the treatment and double-stained with Annexin V-FITC/PI and Annexin V-EnzoGloden/PI for flow cytometric analysis.

Real-time PCR. Total RNAs were extracted with TRIzol reagent for RNA extraction, and the reverse transcription was carried out with RevertAid First-Strand cDNA synthesis kits. Cks1 and Cks2 primers and probes were designed using the ABI Primer Express 2 software. $\beta$-actin was used as the internal loading control. The PCR reactions were performed with the ABI 7500 fluorescent qPCR device.
Western blot analysis. HepG2 cell lysates were prepared by adding $50 \mu \mathrm{l}$ of RIPA lysis buffer to $10^{6}$ of cells. The lysates were separated on SDS-PAGE and transferred to PVDF membranes for western blot analyses. Mouse anti-human $\beta$-actin antibody was used at a 1:1,000 dilution. Rabbit anti-human Cks1 antibody was used at a 1:1,500 dilution. Rabbit anti-human antibody was used at a 1:1,000 dilution. Horseradish peroxidase (HRP)-labeled goat-anti-mouse IgG antibody (targeted to $\beta$-actin) or goat-antirabbit IgG antibody (targeted specifically to Cks1 and Cks2) was used at a 1:10,000 dilution. The specifically bound antibodies were visualized with the ECL chemiluminescence kit.

Statistical analysis. All data were processed with the SPSS 20.0 statistical software package. The overall data were analyzed with Student's t-test. Each assay was repeated in triplicate.

\section{Results}

Depletion of Cks1 and Cks2 expression compromises cell proliferation in HepG2 cells. To assess the role of Cks1/Cks2 in HepG 2 cells, the expression of $\mathrm{Cks} 1 / \mathrm{Cks} 2$ was depleted by siRNA specific to Cks1 and Cks2. The results showed that both Cks1 and Cks2 mRNAs were reduced with $24 \mathrm{~h}$ after the transfection (Fig. 1A). The mRNA levels of Cks1 were downregulated by $95 \%$ and Cks $270 \%$, respectively, at $24 \mathrm{~h}$ after the transfection. The knockdown efficiency gradually decreased over time. Cks1 siRNA maintained a high knockdown efficiency (81\%) even at $96 \mathrm{~h}$ after transfection while the mRNA levels of Cks2 returned to near baseline levels by $96 \mathrm{~h}$ after the transfection. Consistently, expression of Cks1 and Cks2 at the protein level 
A

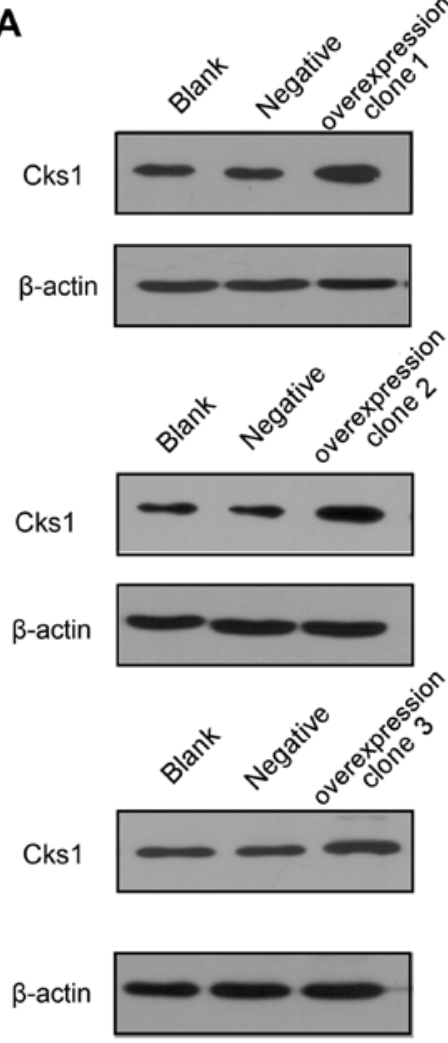

B

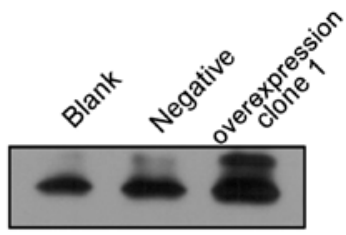

Cks2

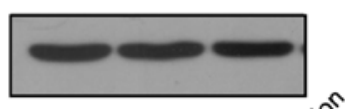

$\beta$-actin

Cks2

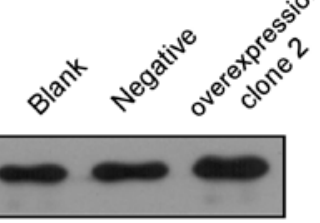

$\beta$-actin
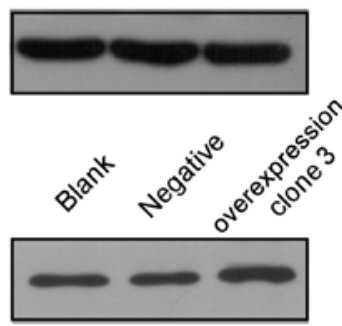

$\beta$-actin

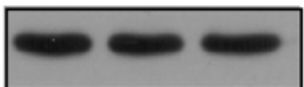

Figure 3. Cks1 and Cks2 protein levels in the overexpressed stable cell lines. (A) Cks1 protein levels in the stable overexpressed cell line. (B) Cks2 protein levels in the stable overexpressed cell line. Overexpression in clones 1-3 represent Cks overexpression of clone 1-3. Negative, negative control, and the blank is untreated HepG2 cells. After transfection of target plasmids, Cks1 and Cks2 protein expression levels were markedly higher in overexpressed cell lines than in the control groups.

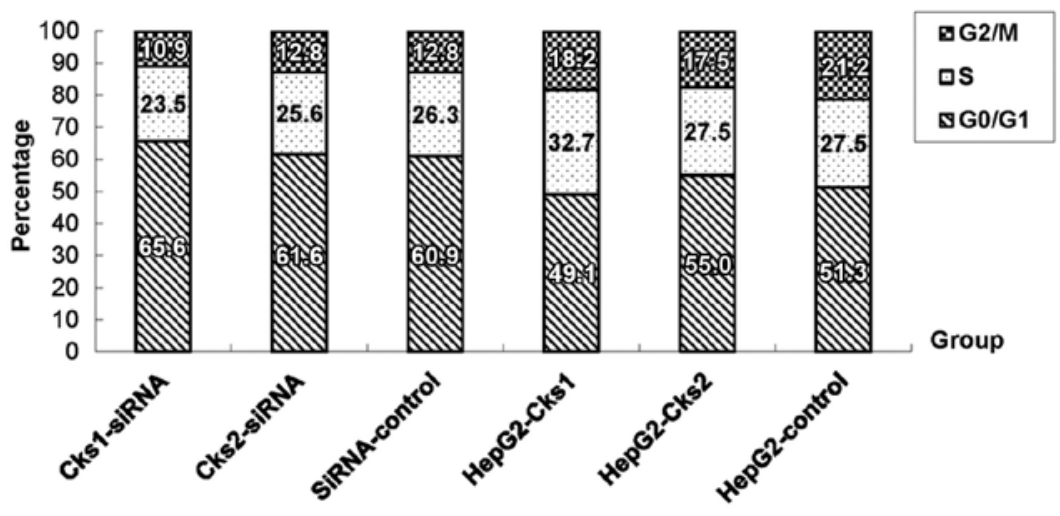

Figure 4. Effect of the changes in Cks1 and Cks2 protein levels on cell cycle in HepG2 cells. The statistical analysis showed that upregulation of Cks1 protein level increased the percentage of cells in $\mathrm{S}$ phase $(\mathrm{P}<0.01)$ and decreased the percentage of cells in $\mathrm{G} 0 / \mathrm{G} 1$ and $\mathrm{G} 2 / \mathrm{M}$ ( $\mathrm{P}<0.01)$ compared to the control. Overexpression of Cks2 only increased the percentage of cells in G0/G1 $(\mathrm{P}<0.05)$ and decreased the percentage of cells in $\mathrm{G} 2 / \mathrm{M}$ ( $\mathrm{P}<0.01)$ compared to the control while the percentage of cells in S phase show no significant difference. The percentage of cells in each phase after Cks1 and Cks2 siRNA transfection was not significantly different from the control.

was also reduced (Fig. 1B). To determine whether depletion of either Cks1 or Cks2 affects cell proliferation, we used the CCK-8 assay kit to examine cell proliferation at $24,48,72$ and $96 \mathrm{~h}$ after transfection with Cks1 and Cks2 siRNA (Fig. 2A). It was clear that cell proliferation was reduced within $24 \mathrm{~h}$ after the transfection. The difference became more obvious over time and remained statistically significant. Quantitation of cell numbers directly with a cell counter also revealed that depletion of Cks1 and Cks2 reduced cell proliferation in HepG2 cells (Fig. 2B). The data suggest that expression of Cks1 and Cks2 has positive effects on cell proliferation. Consistently, overexpression of Cks1 and Cks2 promote cell proliferation (Figs. 3 and $2 \mathrm{C}$ and D). In addition, cell cycle analyses with the fluorescence-assisted cell sorter (FACS) analyses showed that overexpression of Cks1 promoted HepG2 cell transition into S phase (Fig. 4).

Depletion of Cks1 and Cks2 expression enhances chemotherapy agent-induced apoptosis in HepG2 cells. In order to study the effect of $\mathrm{Cks} 1$ and $\mathrm{Cks} 2$ on $\mathrm{HepG} 2$ cellular apoptosis, expression of Cks1 and Cks2 was depleted by 

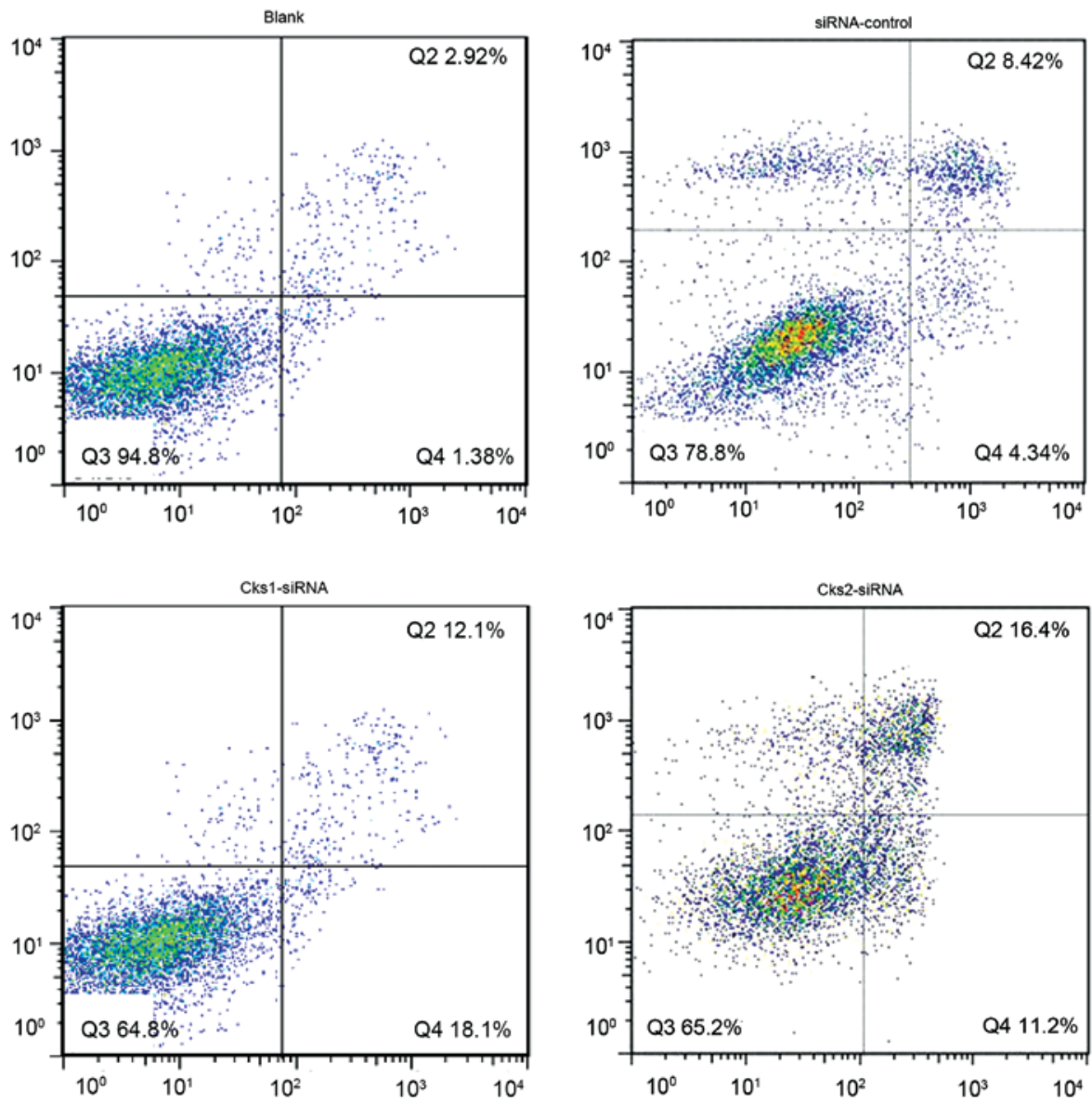

Figure 5. Effect of changes in Cks1 and Cks2 protein levels on the apoptosis rate at early and late stages in HepG2 cells (transient siRNA transfection). The apoptosis rate at early and late stages after transient siRNA transfection.
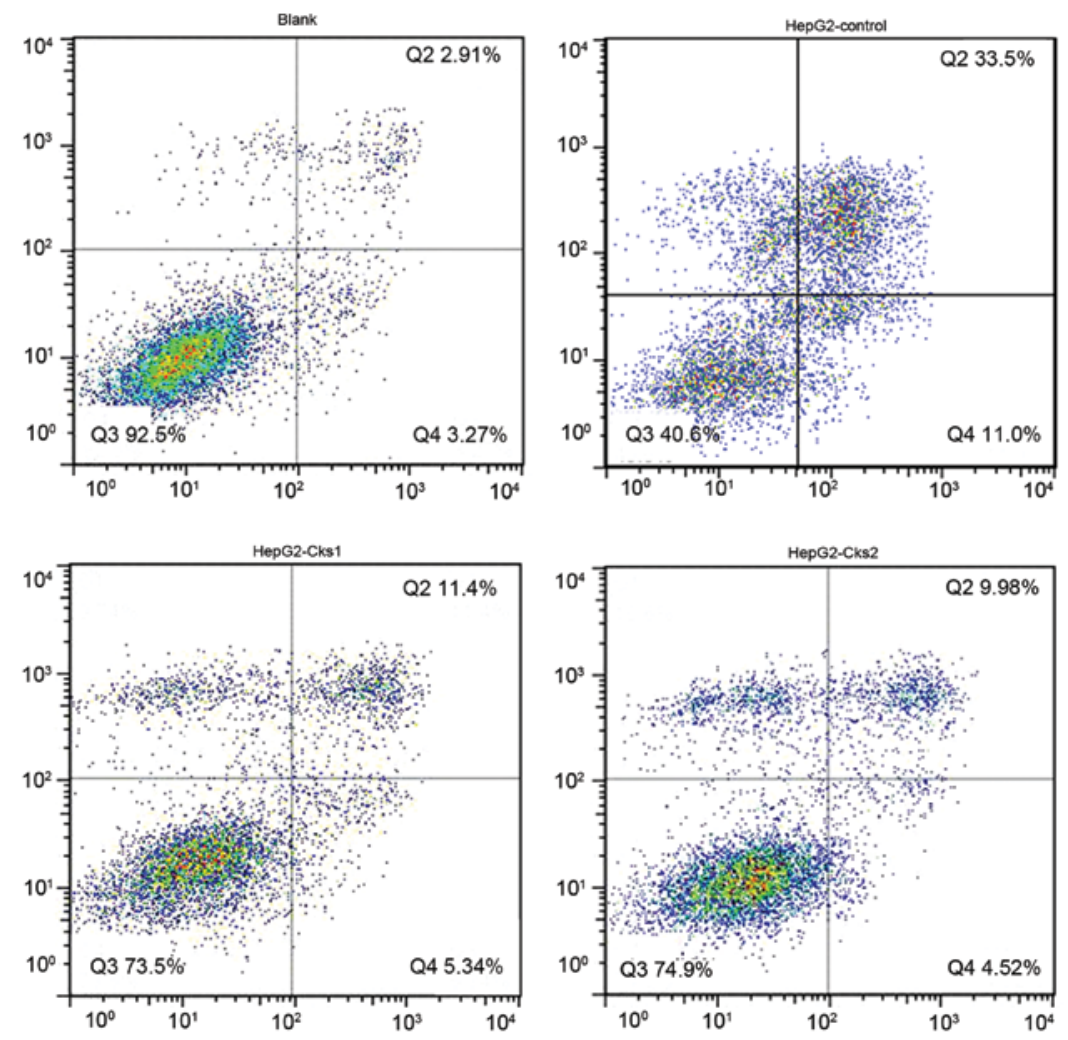

Figure 6. Effect of changes in Cks1 and Cks2 protein levels on the apoptosis rate at early and late stages in HepG2 cells (Cks overexpressed cell lines). The apoptosis rate at early and late stages in Cks overexpressed cell lines. 

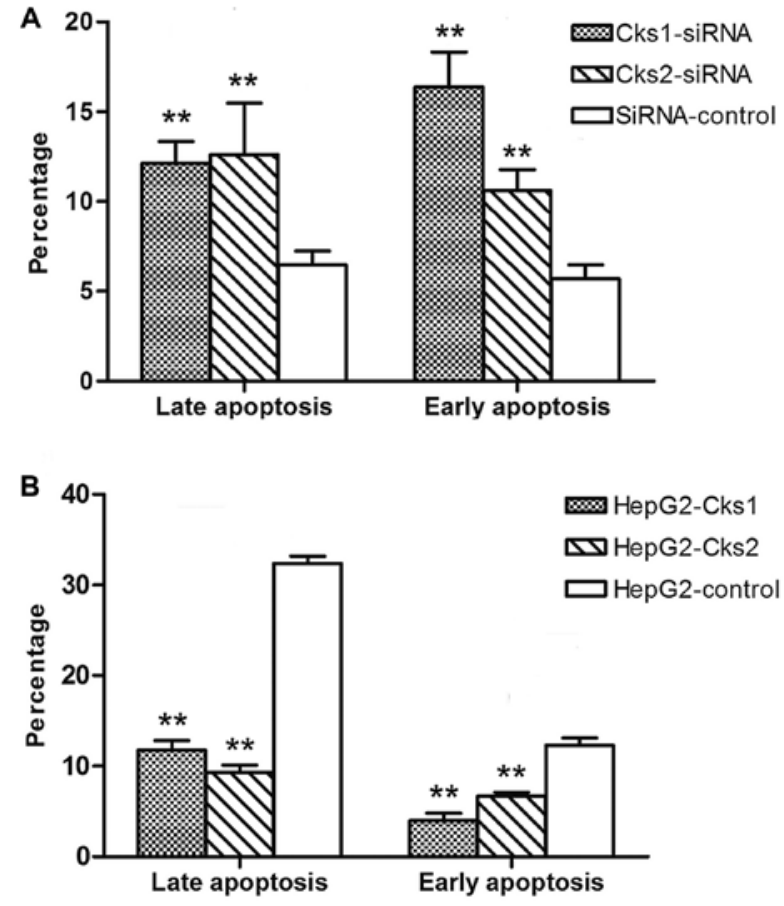

Figure 7. The statistical analysis of apoptosis in HepG2 cells. (A) The apoptosis rate at early and late stages after transient siRNA transfection. (B) The apoptosis rate at early and late stages in Cks overexpressed cell lines. ${ }^{* * *} \mathrm{P}<0.001$

siRNA transfection. Twenty-four hours later, the cells were then treated with cisplatin for another $24 \mathrm{~h}$ followed by Annexin V/PI staining. Flow cytometry was used to analyze the apoptosis rate for both the control and experimental group (Fig. 5). The results indicate that the apoptosis rate in Cks1 and Cks2 knockdown groups was higher than that in the control group upon cisplatin treatment at both early and late stages $(\mathrm{P}<0.01$, Fig. 7A). Consistently, overexpression of Cks1 and $\mathrm{Cks} 2$ resulted in a lower cisplatin-induced apoptosis rate at both the early and the late stages as compared with the control $(\mathrm{P}<0.01)$ (Figs. 6 and 7B). Together, the results demonstrate that high expression level of $\mathrm{Cks} 1$ and $\mathrm{Cks} 2$ protects the cells from undergoing apoptosis upon chemotherapy drug treatment.

Depletion of either Cks1 or Cks2 promotes AKT and GSK-3 $\beta$ phosphorylation in Hep 2 cells. To investigate the mechanism by which depletion of Cks1 and Cks2 affects cell apoptosis, we then assessed activation of AKT and GSK activation in Cks depleted cells by western blot analyses. The results revealed that depletion of either Cks1 or Cks2 reduced AKT and GSK-3 $\beta$ phosphorylation (Fig. 8). Since activity of AKT and Wnt signaling has been shown to suppress cell apoptosis, the results suggested that high expression of $\mathrm{Cks}$ proteins protects HCC cells from apoptosis via the AKT and Wnt pathways.

\section{Discussion}

Currently, only a limited number of cancer targeting drugs are available for HCC treatment (9). However, liver cancer frequently develops tolerance to the currently available molecular targeting drugs $(10,11)$. Thus, it is imperative to develop new targeting drugs for HCC. In the present study,
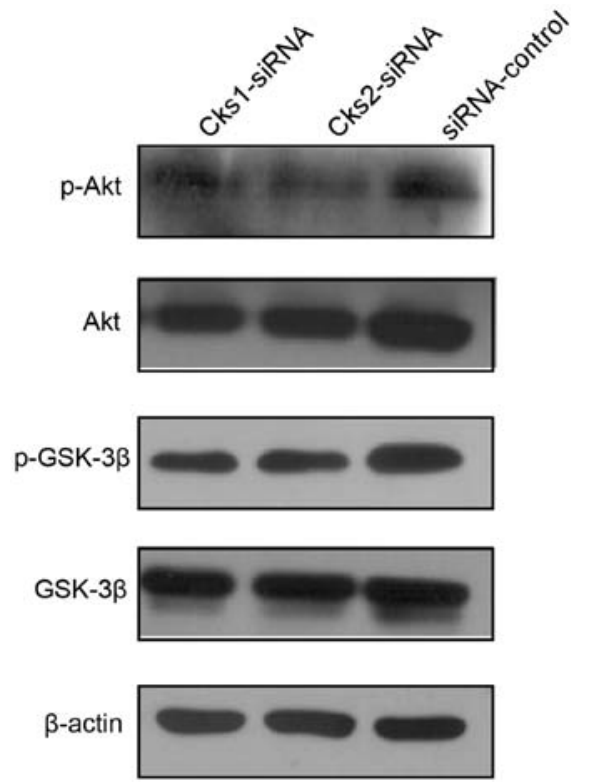

Figure 8. The effect of changes in Cks1 and Cks2 protein levels on Akt and GSK-3 $\beta$. Compared to the control group, the expression of Akt and GSK-3 $\beta$ was not significantly affected by Cks1 and Cks2 knockdown. However, phosphorylation of Akt and GSK-3 $\beta$ was significantly reduced by both Cks1 and Cks2 siRNA interference, which suggested that Cks1 and Cks2 potentially regulate cellular proliferation and apoptosis through activation of Akt/GSK$3 \beta$-related signaling pathways.

we described that depleting Cks1 and Cks2 in HepG2 cells reduces cell proliferation and increase apoptosis induced by the common chemotherapy reagents. The results suggest that targeting Cks1 and Cks2 in conjunction with chemotherapy treatment can be a more effective therapy for HCC.

Although Cks1 promoted p27 ubiquitination and degradation (12), and therefore, promotes cell proliferation, the function of Cks2 is largely unknown in somatic cells. Furthermore, how depletion of $\mathrm{Cks} 1$ and $\mathrm{Cks} 2$ increase sensitivity to cancer chemotherapy drugs are still not clear. Further efforts are needed to characterize the molecular mechanism by which Cks1 and Cks2 protect cells from undergoing apoptosis upon chemotherapy treatment. The caspase family of proteins play a critical role in mediating cellular apoptosis. It has been reported that $\mathrm{Cks} 2$ downregulation reduces cell proliferation and increase caspase-3 activation and Bax expression in gastric cancer cells (6). Depleting Cks2 expression with siRNA in esophageal squamous cell carcinoma also results in reducing cell proliferation (13).

Activation of Akt via phosphorylation plays an important role in cell survival and apoptosis (14-16). It suppresses apoptosis and promotes cell survival, and regulates glycogen synthesis via GSK-3 $\beta$ and the cell cycle by blocking GSK-3 $\beta$-mediated cyclin D phosphorylation and degradation (17). In addition, Akt plays a key role in cell proliferation. Our results showed that downregulation of $\mathrm{Cks} 1$ and $\mathrm{Cks} 2$ protein expression led to a decrease in Akt and GSK-3 $\beta$ phosphorylation. It is speculated that the Cks1- and Cks2-regulated Akt and GSK-3 $\beta$ signaling activity underlies the oncogenic activities of both Cks1 and Cks2.

In summary, Cks1 and Cks2 promote HCC cell proliferation and suppress HCC cell apoptosis induced by chemotherapy drugs. Therefore, suppressing Cks1 and Cks2 activity likely 
will increase efficacy of chemotherapy for HCC, which deserves further investigation.

\section{Acknowledgements}

We appreciate the support of all the staff at the Central Laboratory of Xiamen University Affiliated Zhongshan Hospital during the conduct of the experiments. In addition, the cell lines that we used in this study were generously provided by the Hepatobiliary Surgery Laboratory at the Xiamen University Affiliated Zhongshan Hospital. This study was supported by the National Natural Science Foundation of China (Grant No. 81072016).

\section{References}

1. Richardson HE, Stueland CS, Thomas J, Russell P and Reed SI: Human cDNAs encoding homologs of the small p34Cdc28/Cdc2-associated protein of Saccharomyces cerevisiae and Schizosaccharomyces pombe. Genes Dev 4: 1332-1344, 1990.

2. Wang JJ, Fang ZX, Ye HM, You P, Cai MJ, Duan HB, Wang $F$ and Zhang ZY: Clinical significance of overexpressed cyclin-dependent kinase subunits 1 and 2 in esophageal carcinoma. Dis Esophagus 26: 729-736, 2013.

3. Lan Y, Zhang Y, Wang J, Lin C, Ittmann MM and Wang F: Aberrant expression of Cks1 and Cks2 contributes to prostate tumorigenesis by promoting proliferation and inhibiting programmed cell death. Int J Cancer 123: 543-551, 2008.

4. Yu M, Zhong M and Qiao Z: Expression and clinical significance of cyclin kinase subunit 2 in colorectal cancer. Oncol Lett 6 777-780, 2013

5. Lee SW, Kang SB, Lee DS and Lee JU: Akt and Cks1 are related with lymph node metastasis in gastric adenocarcinoma. Hepatogastroenterology 60: 932-937, 2013.

6. Tanaka F, Matsuzaki S, Mimori K, Kita Y, Inoue H and Mori M: Clinicopathological and biological significance of CDC28 protein kinase regulatory subunit 2 overexpression in human gastric cancer. Int J Oncol 39: 361-372, 2011.
7. Shen DY,Zhan YH, Wang QM, Rui G and Zhang ZM: Oncogenic potential of cyclin kinase subunit-2 in cholangiocarcinoma. Liver Int 33: 137-148, 2013.

8. Shen DY, Fang ZX, You P, Liu PG, Wang F, Huang CL, Yao XB, Chen ZX and Zhang ZY: Clinical significance and expression of cyclin kinase subunits 1 and 2 in hepatocellular carcinoma. Liver Int 30: 119-125, 2010.

9. Li F, Zhao C and Wang L: Molecular-targeted agents combination therapy for cancer: Developments and potentials. Int $\mathbf{J}$ Cancer 134: 1257-1269, 2014.

10. Ricke J, Bulla K, Kolligs F, Peck-Radosavljevic M, Reimer P, Sangro B, Schott E, Schütte K, Verslype C, Walecki J, et al; SORAMIC study group: Safety and toxicity of radioembolization plus sorafenib in advanced hepatocellular carcinoma: Analysis of the European multicentre trial SORAMIC. Liver Int 35: 620-626, 2015.

11. Zhu AX, Duda DG, Ancukiewicz M, di Tomaso E, Clark JW, Miksad R, Fuchs CS, Ryan DP and Jain RK: Exploratory analysis of early toxicity of sunitinib in advanced hepatocellular carcinoma patients: Kinetics and potential biomarker value. Clin Cancer Res 17: 918-927, 2011.

12. Ganoth D, Bornstein G, Ko TK, Larsen B, Tyers M, Pagano M and Hershko A: The cell-cycle regulatory protein Cks1 is required for $\mathrm{SCF}(\mathrm{Skp} 2)$-mediated ubiquitinylation of $\mathrm{p} 27$. Nat Cell Biol 3: 321-324, 2001.

13. Kita Y, Nishizono Y, Okumura H, Uchikado Y, Sasaki K, Matsumoto M, Setoyama T, Tanoue K, Omoto I, Mori S, et al: Clinical and biological impact of cyclin-dependent kinase subunit 2 in esophageal squamous cell carcinoma. Oncol Rep 31: 1986-1992, 2014.

14. Franke TF, Kaplan DR and Cantley LC: PI3K: Downstream AKTion blocks apoptosis. Cell 88: 435-437, 1997.

15. Burgering BM and Coffer PJ: Protein kinase B (c-Akt) in phosphatidylinositol-3-OH kinase signal transduction. Nature 376: 599-602, 1995

16. Franke TF, Yang SI, Chan TO, Datta K, Kazlauskas A, Morrison DK, Kaplan DR and Tsichlis PN: The protein kinase encoded by the Akt proto-oncogene is a target of the PDGF-activated phosphatidylinositol 3-kinase. Cell 81: 727-736, 1995.

17. Hajduch E, Litherland GJ and Hundal HS: Protein kinase B (PKB/Akt) - a key regulator of glucose transport? FEBS Lett 492: 199-203, 2001. 